\title{
Avaliação andrológica por pontos e comportamento sexual em touros da raça Nelore
}

\section{Flávio Guiselli Lopes ${ }^{1}$, José Domingos Guimarães ${ }^{2}$, Eduardo Paulino da Costa ${ }^{2}$, Giovanni Ribeiro de Carvalho ${ }^{3}$, Tamires Miranda Neto ${ }^{4}$}

${ }^{1}$ Doutorando em Veterinária, DVT/UFV - Viçosa - MG.

2 Departamento de Veterinária/UFV - Viçosa - MG.

${ }^{3}$ Departamento de Zootecnia/UFV - Viçosa - MG.

${ }^{4}$ Agropecuária CFM Ltda - São José do Rio Preto - MG.

RESUMO - Objetivou-se estudar a relação entre dois métodos de avaliação do potencial reprodutivo - Classificação Andrológica por Pontos (CAP) e comportamento sexual de touros da raça Nelore - e verificar a acurácia desses testes em predizer o potencial reprodutivo dos touros, avaliado pela taxa de gestação ao final da estação de monta, com duração média de 90 dias. Foram utilizados 14 touros, todos classificados como aptos à reprodução, após exame andrológico, CAP e observação do comportamento sexual a campo durante 12 horas. Onze touros foram avaliados em manejo individual, com proporção média touro:vaca de 1:46, e os três restantes, em manejo múltiplo, com proporção média touro:vaca de 1:23. A pontuação média obtida pela CAP foi 78,92 \pm 8,92 (muito bom) e o escore, 9,85 \pm 0,36 (excelente) para libido. Quanto maior o tempo de teste, maiores foram os escores da libido. Na primeira hora de observação, os touros foram classificados como de libido questionável e ao final do teste, como de libido excelente. As correlações da libido com circunferência escrotal (CE), características físicas e morfológicas do sêmen e taxa de gestação foram baixas. A pontuação pela CAP correlacionou-se positivamente com circunferência escrotal e características físicas do sêmen, mas não se correlacionou com características morfológicas dos sêmen. Não foi observada correlação entre CAP e taxa de gestação, nem entre libido e CAP. A taxa geral de gestação após o período de estação de monta foi de 74,69\%. Os resultados indicam que tanto a CAP como o teste da libido não são eficientes para predizer o potencial reprodutivo de touros da raça Nelore e devem ser utilizados de forma complementar ao exame andrológico.

Palavras-chave: Bos taurus indicus, fertilidade, libido, sêmen, zebuínos

\section{Andrologic evaluation by points and sexual behavior in Nelore bulls}

\begin{abstract}
The objective of this work was to study the relation between two reproductive potential evaluation methods - Andrologic Evaluation by Points - CAP and sexual behavior of Nelore bulls, and to verify the accuracy of these tests in predicting the reproductive potential of bulls through the gestation rate at the end of the breeding season, with average duration of ninety days. Fourteen bulls were used, all classified as able for reproduction, evaluated through andrologic examination, being submitted to CAP and observation of the sexual behavior at field (libido test) during 12 hours. Eleven bulls were evaluated in individual handling, with an average bull:cow ratio of 1:46 and the rest of the animals (three), evaluated in a multiple handling, with an average bull:cow ratio of 1:23. The average score obtained by CAP was $78.92 \pm 8.92$ (very good) and the score of $9.85 \pm 0.36$ (excellent) for the libido test. The longer the test was, the higher libido scores were obtained. At the first time of observation, the bulls were classified as with questionable libido and at the end of the test as with excellent libido. The correlations between libido, scrotal circumference (SC), physical and morphological characteristics of the semen and the gestation rate were low. The scores obtained through CAP was positively correlated with SC and physical characteristics of the semen; however, no correlation was observed for the morphologic characteristics. No correlation was observed between CAP and gestation rate or between libido and CAP. The overall gestation rate after the breeding period was $74.69 \%$. The results suggest that both CAP and the libido test were not efficient to predict the reproductive potential of Nelore bulls and should be used as a complement to the andrologic evaluation.
\end{abstract}

Key Words: Bos taurus indicus, fertility, libido, semen, zebu 


\section{Introdução}

A pecuária brasileira possui um rebanho comercial bovino estimado 200 milhões de cabeças (IBGE, 2009) constituído de $80 \%$ de animais das raças indianas e seus mestiços (Anualpec, 2003). Considerando a baixa taxa de fertilidade e desfrute registrada no País, com número de animais inférteis e subférteis em serviço de aproximadamente 40\% (Sereno, 2004) e regime de monta natural como principal manejo reprodutivo (95\%) (Fonseca, 2000), o touro é fundamental na eficiência reprodutiva e produtiva do rebanho.

Segundo Fonseca et al. (1991), a utilização inadequada do potencial reprodutivo dos machos aumenta a demanda de touros, ocasionando a utilização de reprodutores sem seleção genética prévia para características morfológicas e funcionais, como fertilidade, precocidade ponderal e/ou sexual e habilidade materna. Dessa forma, as características físicas e morfológicas do sêmen, a biometria testicular, o desenvolvimento ponderal e o comportamento sexual devem fazer parte do exame andrológico para predição do potencial reprodutivo dos touros.

Nesse contexto, vários pesquisadores contribuíram para a evolução do processo de diagnóstico e classificação de touros. Entre os padrões preconizados para avaliação da capacidade reprodutiva de touros europeus, o mais difundido tem sido o padrão norte-americano Breeding Soundness Evaluation - BSE, proposto por Chenoweth \& Ball (1980). Nesse sistema, os animais são avaliados de acordo com a circunferência escrotal em cada faixa etária e também com aspectos físicos e morfológicos do sêmen.

No Brasil, para avaliação, classificação e seleção de touros de raças zebuínas, várias tabelas têm sido propostas com algumas adaptações, principalmente relacionadas à idade e à circunferência escrotal (Fonseca et al., 1997a). Atualmente, o Colégio Brasileiro de Reprodução Animal (CBRA, 1998) preconiza duas tabelas de pontuação: uma instituída por Fonseca et al. (1997a) para touros zebuínos (Bos taurus indicus) e outra para touros europeus (Bos taurus taurus), segundo padrões preconizados por Chenoweth \& Ball (1980).

Para avaliação do comportamento sexual de touros, Pineda et al. (2000a) e Santos et al. (2004b) sugerem a utilização de testes da libido como fator de seleção para animais com melhor desempenho reprodutivo, visando aumentar os índices de fertilidade de rebanhos, auxiliar na determinação da proporção touro:vaca, reduzir os custos na aquisição de novos animais e aumentar a produtividade.

O comportamento sexual depende de uma interação sócio-sexual que conjuga fatores ambientais, nutricionais, hormonais, freqüência de acasalamentos, receptividade do heterossexo, acuidade sensorial, idade, experiência prévia do indivíduo e hierarquia social, além de fatores genéticos. O comportamento sexual do macho está associado à detecção do estro e à capacidade de cópula com uma fêmea, que envolve basicamente a libido e a capacidade de serviço (Chenoweth, 1983).

Neste sentido, o objetivo com este trabalho foi estudar a relação entre dois métodos de avaliação do potencial reprodutivo - Classificação Andrológica por Pontos (CAP) e comportamento sexual-de touros da raça Nelore e verificar a acurácia desses testes em predizer o potencial reprodutivo e a taxa de gestação no final da estação de monta.

\section{Material e Métodos}

Quatorze touros da raça Nelore (Bos taurus indicus), clinicamente normais, com 36 a 96 meses de idade, foram submetidos a exame andrológico completo (avaliação física e morfológica do sêmen ebiometria testicular), a classificação andrológica por pontos e à observação do comportamento sexual a campo. Todos os animais se encontravam em boa condição corporal e com média de escore 6 (escala de 1 a 9) (Lôbo, 2001). O sistema de manejo dos touros no período entre estações reprodutivas foi extensivo, em regime de pasto, com predominância de capim-braquiária (Brachiaria decumbens), sem rotação de piquetes e fornecimento de água e sal mineral à vontade.

O potencial reprodutivo dos machos foi verificado em estação de monta, com duração média de 90 dias para cada lote, e fornecimento de água e sal mineral à vontade. Foram utilizadas pastagens planas, cultivadas com capimbraquiária (Brachiaria decumbens), colonião (Panicum maximun) e tobiatã (Panicum maximun cv), situadas em solos de mesma qualidade, com taxa de lotação de aproximadamente 1 UA/ha. As 609 vacas da raça Nelore utilizadas estavam com 4 anos de idade, intervalo de partos regular de 14 meses, sem perdas de crias e último parto entre os meses de setembro e dezembro de 2002, todas em boa condição corporal.

As medições da circunferência escrotal foram realizadas com auxílio de fita métrica milimetrada, posicionada na região mediana do escroto, no ponto de maior dimensão, envolvendo as duas gônadas e a pele escrotal. As mensurações, em centímetros, do comprimento (sentido proximal-distal, incluindo cabeça e excluindo a cauda do epidídimo) e da largura testicular (sentido médio-lateral, no ponto de maior diâmetro) foram obtidas com auxílio de paquímetro.

O sêmen foi obtido pelo método da eletroejaculação, de modo que os ejaculados foram coletados em tubos coletores 
graduados, acoplados a funis plásticos previamente aquecidos em estufa mantida a $37^{\circ} \mathrm{C}$. O sêmen coletado foi imediatamente avaliado conforme metodologia proposta por Fonseca et al. (1992) para avaliação do turbilhonamento, da motilidade espermática progressiva retilínea e do vigor espermático. O turbilhonamento (movimento em massa) foi avaliado com auxílio de um microscópio binocular, em aumento de 100x. Para isso, uma gota de sêmen foi colocada sobre uma lâmina previamente aquecida a $37^{\circ} \mathrm{C}$ e classificada de acordo com o movimento espermático, em escala de 0 a 5. Posteriormente, utilizando nova alíquota de sêmen entre lâmina e lamínula, previamente aquecidas a $37^{\circ} \mathrm{C}$, sob aumento de $400 \mathrm{x}$, foram classificados a motilidade espermática progressiva retilínea (percentual de espermatozóides com movimento progressivo retilíneo) e o vigor espermático (intensidade do movimento dos espermatozóides), avaliado em escala de 0 a 5. Para avaliação das características morfológicas dos espermatozóides, uma alíquota de sêmen de cada ejaculado foi acondicionada em ependorff contendo 1,0 mL de solução formol salina tamponada (Hancock, 1957), em quantidade necessária para turvar a amostra, e estocada em temperatura ambiente por período máximo de um mês. As análises morfológicas do sêmen foram feitas em preparações úmidas sob imersão, em microscopia de contraste de fase, com aumento de $1000 x$, com avaliação de 400 células por ejaculado. As anomalias espermáticas foram computadas conforme classificação de Blom (1983) quanto aos defeitos espermáticos maiores e defeitos espermáticos totais.

Após a avaliação andrológica, os touros foram classificados quanto aos seguintes parâmetros: circunferência escrotal - até 40 pontos; aspectos físicos (motilidade espermática progressiva retilínea e vigor espermático) - até 25 pontos; morfologia espermática (defeitos maiores e totais) - até 35 pontos, que, somados, permitiram obter a classificação final em questionáveis, bons, muito bons e excelentes (Fonseca et al., 1997a) (Tabela 1).

Os touros foram observados durante a estação reprodutiva da fazenda (15/11/2002 a 15/1/2003), de modo que 11 foram avaliados em manejo individual, com proporção média touro:vaca de 1:46, e os três restantes, em manejo múltiplo, com proporção média touro:vaca de 1:23.

O comportamento sexual foi observado segundo o Método do Animal Focal (Altmann, 1974), de modo que os touros foram foco principal durante todo o período de observação. As observações foram feitas somente no período diurno (das 6 às 18 h, horário de Brasília), totalizando 12 horas de teste, subdivididas em quatro períodos de 3 horas: período 1 = horário de observação das 6 às $9 \mathrm{~h}$; período 2 = horário de observação das 9 às 12 h; período $3=$ horário de observação das 12 às 15 h; e período 4 = horário de observação das 15 às $18 \mathrm{~h}$. Esses períodos foram agrupados de acordo com a temperatura ambiente, o que possibilitou separar os períodos de temperaturas mais elevadas

Tabela 1 - Classificação andrológica por pontos sugerida para touros da subespécie Bos taurus indicus, baseada na circunferência escrotal e nas características físicas e morfológicas do sêmen

\begin{tabular}{|c|c|c|c|c|}
\hline \multirow[t]{2}{*}{ Parâmetro } & \multicolumn{4}{|c|}{ Classificação } \\
\hline & Excelente & Muito bom & Bom & Questionável \\
\hline \multicolumn{5}{|l|}{ Motilidade espermática } \\
\hline Vigor $(0-5)$ & 5 & $4<5$ & $3<4$ & $<3$ \\
\hline Motilidade progressiva (\%) & 75 & $60-75$ & $30<60$ & $<30$ \\
\hline Pontos outorgados & $21-25$ & $16<21$ & $10<16$ & $<10$ \\
\hline \multicolumn{5}{|l|}{ Morfologia espermática } \\
\hline Defeitos maiores (\%) & 5 & $>5-10$ & $>10-20$ & $>20$ \\
\hline Defeitos totais (\%) & 10 & $>10-15$ & $>15-30$ & $>30$ \\
\hline Pontos outorgados & $30-35$ & $25<30$ & $15<25$ & $<15$ \\
\hline \multicolumn{5}{|l|}{ Circunferência escrotal (cm) } \\
\hline \multicolumn{5}{|l|}{ Idade em meses } \\
\hline $7-12$ & 21,0 & $19,5<21,0$ & $17,5<19,5$ & $<17,5$ \\
\hline $12-18$ & 26,0 & $24,0<26,0$ & $21,5<24,0$ & $<21,5$ \\
\hline $18-24$ & 31,5 & $28,5<31,5$ & $26,0<28,5$ & $<26,0$ \\
\hline $24-36$ & 35,0 & $32,0<35,0$ & $29,0<32,0$ & $<29,0$ \\
\hline $36-48$ & 37,0 & $33,5<37,0$ & $30,5<33,5$ & $<30,5$ \\
\hline$>48$ & 39,0 & $36,0<39,0$ & $33,0<36,0$ & $<33,0$ \\
\hline Pontos outorgados & $35-40$ & $25<35$ & $15<25<15$ & \\
\hline Total de pontos do reprodutor & $86-100$ & $66<86$ & $40<66$ & $<40$ \\
\hline
\end{tabular}

Fonte: Fonseca et al. (1997a). 
(9 às 12 h e 12 às 15 h) das temperaturas mais amenas do dia (6 às 9 h e 15 às 18 h). Todos os movimentos do macho diante de fêmeas em estro natural foram anotados, com o horário de ocorrência, e os dados foram posteriormente incluídos na planilha de eventos fisiológicos preconizada por Santos et al. (2004b).

Para iniciar o período de observação, realizou-se o rodeio do rebanho, em determinado piquete, com o objetivo de facilitar o encontro dos parceiros sexuais. Após a ocorrência do estro (receptividade ao macho ou fêmeas do grupo), um observador a cavalo procedeu às observações, com auxílio de um binóculo, com distanciamento para reduzir o efeito de sua presença no comportamento dos animais.

Os movimentos relacionados ao comportamento sexual do macho diante da fêmea em estro foram agrupados em quatro classes, segundo classificação proposta por Costa e Silva (2004) como: comportamentos de identificação cheirada e lambida de corpo (CC), cheirada e lambida de vulva (CV), reflexo de Flehmen (RF); comportamentos précopulatórios - reflexo de monta (RM), exposição de pênis (EP), monta sem exposição de pênis (MSEP), tentativa de monta (TM) e monta abortada (MA); comportamento copulatório-serviço completo (SC) e outros comportamentos - acompanhamento de fêmea (AF), pressão no cupim (PC), frente a frente $(\mathrm{FF})$.

Posteriormente, de acordo com os eventos fisiológicos, os reprodutores foram classificados de acordo com a libido, segundo Tabela proposta pelo Colégio Brasileiro de Reprodução Animal (CBRA, 1998) para classificação da libido nos testes convencionais de 10 minutos. Neste estudo, foram considerados os eventos fisiológicos dos animais no período de 1 hora, que foram classificados em cada hora, durante as 12 horas de observação. Os reprodutores também foram classificados considerando todos os eventos fisiológicos durante o período de observação de forma acumulativa.

Os diagnósticos de gestação foram realizados 30 dias após o término da estação de monta, por meio de exame ultra-sonográfico (Ultra-som Aloka, modelo SSD-500, acoplado a um transdutor transretal linear de 5,0 MHz). As fêmeas foram consideradas gestantes quando visualizada a vesícula embrionária ou o próprio embrião.

Utilizou-se o programa estatístico SAEG (UFV, 2002) para a análise estatística dos dados, com análises descritivas (média, desvio-padrão, coeficiente de variação, amplitude e distribuição de freqüência) de todas as variáveis estudadas. Correlações simples de Pearson foram calculadas entre todos os parâmetros estudados, principalmente os escores de pontuação alcançados pelos reprodutores nos dois testes propostos (CAP e comportamento sexual). Para avaliação do comportamento das características quantitativas em cada horário de observação, aplicou-se análise de regressão.

As características qualitativas (taxa de gestação) foram analisadas por avaliação dos dados em tabelas de contingência e, posteriormente, pelo teste do Qui-quadrado, com $5 \%$ de probabilidade de erro, para verificar se houve efeito do touro sobre a taxa de gestação alcançada ao final da estação de monta. Os dados referentes à freqüência dos eventos fisiológicos e/ou às classes de comportamento sexual em cada período (manhã, tarde, 6 às 9 h, 9 às 12 h, 12 às 15 h e 15 às 18 h) também foram agrupados em tabelas de contingência e analisados pelo teste do Qui-quadrado, com 5\% de probabilidade de erro.

\section{Resultados e Discussão}

O valor médio de circunferência escrotal foi de 37,75 \pm $1,50 \mathrm{~cm}$ (Tabela 2), muito próximo daquele verificado por Costa \& Silva (1994), 37,87 $\pm 2,60$ cm, também em touros Nelore com idade superior a 5 anos, e ao reportado por Gottschall \& Mattos (1997), 37,0 $\pm 2,0 \mathrm{~cm}$, e Santos et al. (2004a), 37,18 $\pm 2,02 \mathrm{~cm}$, em touros de mesma raça e faixa etária (cinco anos). Esse resultado, no entanto, foi superior ao registrado por Pineda et al. (1997), 36,36 $\pm 0,35 \mathrm{~cm}$, e Pineda et al. (2000b), 36,29 $\pm 2,40 \mathrm{~cm}$, em touros Nelore com média de 72 meses de idade.

Considerando a tabela proposta por Fonseca et al. (1997a) e pelo Colégio Brasileiro de Reprodução Animal (CBRA, 1998) para classificação de touros Nelore quanto à circunferência escrotal, os touros utilizados nesta pesquisa podem ser classificados como muito bons.

Os valores médios obtidos para turbilhonamento, motilidade espermática progressiva retilínea e vigor espermático enquadram-se nos padrões preconizados por Fonseca et al. (1992) e pelo Colégio Brasileiro de Reprodução Animal (CBRA, 1998). As médias para motilidade espermática progressiva retilínea e vigor espermático foram de $74,28 \pm 12,38 \%$ e $3,0 \pm 0,94$, que correspondem, respectivamente, a muito bom e bom, de acordo com Fonseca et al. (1997a). As médias obtidas para defeitos maiores (5,89\%) e defeitos totais (9,5\%) estão dentro dos limites preconizados por Fonseca et al. (1992) e pelo Colégio Brasileiro de Reprodução Animal (CBRA, 1998) para classificação, respectivamente, como muito bom e excelente (Fonseca et al., 1997a).

A classificação andrológica por pontos para os touros foi feita de acordo com a metodologia preconizada por Fonseca et al. (1997a) e os resultados permitiram classificar 
Tabela 2 - Características reprodutivas de touros Nelore

\begin{tabular}{|c|c|c|c|c|}
\hline Característica & Média \pm desvio-padrão & Coeficiente de variação & Mínimo & Máximo \\
\hline Circunferência escrotal (cm) & $37,75 \pm 1,50$ & 4,0 & 32,3 & 47,6 \\
\hline Libido $(0-10)$ & $9,85 \pm 0,36$ & 3,7 & 09 & 10 \\
\hline Classificação andrológica por pontos (0-100) & $78,92 \pm 8,92$ & 11,3 & 63 & 100 \\
\hline Turbilhonamento (0-5) & $2,55 \pm 1,33$ & 52,2 & 0 & 4 \\
\hline Vigor $(0-5)$ & $3,00 \pm 0,94$ & 31,3 & 2 & 5 \\
\hline Motilidade (\%) & $74,28 \pm 12,38$ & 16,7 & 50 & 90 \\
\hline
\end{tabular}

os touros como muito bons $(78,92 \pm 8,92)$. O teste da libido, com duração de 12 horas, foi executado a campo, utilizando-se a metodologia proposta por Pineda et al. (1997), e os touros foram classificados como excelentes $(9,85 \pm 0,36)$. Os resultados obtidos neste estudo foram superiores aos descritos por diversos autores, como Pineda et al. (1997), Pineda et al. (2000a), Pineda et al. (2000b) e Sarreiro et al. (2002), em touros Nelore avaliados em curral durante dez minutos.

As estimativas de correlações simples de Pearson da libido com cinscunferência escrotal, características físicas e morfológicas foram baixas ( $\mathrm{P}>0,05)$. No entanto, foram observadas correlações $(\mathrm{P}<0,05)$ entre libido e turbilhonamento $(r=0,63)$ e entre libido e circunferência escrotal $(\mathrm{r}=0,51)$. Em razão do pequeno número de touros avaliados, essas correlações não foram consideradas representativas e apresentaram dispersão muito grande dos dados em torno da reta. Galvani (1998), em experimento com 170 touros Nelore, com média de 72 meses de idade, verificou correlações negativas, próximas de zero e não-significativas, entre libido e características físicas e morfológicas do sêmen. Resultados semelhantes foram descritos por Pineda \& Lemos (1994), Pineda et al. (1997), Sarreiro et al. (2000) e Oliveira et al. (2007).

As equações de regressão que melhor explicaram a relação entre libido e turbilhonamento e entre libido e circunferência escrotal foram lineares: ( $\hat{Y}=9,46+0,16 x$, $\left.\mathrm{R}^{2}=0,40, \mathrm{P}<0,05\right)$ e $\left(\hat{\mathrm{Y}}=7,95+0,05 \mathrm{x}, \mathrm{R}^{2}=0,26, \mathrm{P}<0,05\right)$, respectivamente.

Neste estudo não foi observada correlação entre libido e classificação andrológica por pontos $(\mathrm{P}>0,05)$, o que indica que os dois métodos de avaliação são independentes e que classificação andrológica por pontos, circunferência escrotal e características físicas e morfológicas do sêmen não se correlacionam com libido $(\mathrm{P}>0,05)$, portanto, o teste da libido deve ser considerado um método de avaliação complementar ao exame andrológico, que não pode ser utilizado de forma isolada.
Entretanto, classificação andrológica por pontos (CAP) correlacionou-se positivamente com circunferência escrotal e com características físicas do sêmen $(\mathrm{P}<0,05)$. As correlações foram de 0,65 entre CAP e circunferência escrotal; 0,61 entre CAP e vigor espermático; e 0,48 entre CAP e motilidade espermática progressiva retilínea. Para as características morfológicas do sêmen, no entanto, não foram observadas correlações significativas $(\mathrm{P}>0,05)$. Freneau et al. (1996), Freneau et al. (1998), Quirino (1998), Ribeiro Filho et al. (1998), Freneau et al. (2000), Andrade et al. (2001) e Salvador et al. (2001) observaram correlações negativas $(\mathrm{P}<0,05)$ entre CAP e características morfológicas do sêmen.

A ausência de correlação entre CAP e características morfológicas do sêmen $(\mathrm{P}<0,05)$ provavelmente foi ocasionada pelo pequeno número de animais utilizados no experimento, visto que, em estudos realizados por outros autores, o número de animais utilizados foi muito superior. A correlação entre CAP e taxa de gestação foi negativa $(r=-0,61)(P<0,05)$. Resultados semelhantes foram reportados por Costa e Silva et al. (1990) e Costa e Silva (1994), que, em pesquisa com touros adultos Nelore, observaram que CAP não influenciou a taxa de gestação média durante a estação de monta e não teve efeito significativo sobre a fertilidade.

A correlação $(\mathrm{P}>0,05)$ entre libido e taxa de gestação deve-se, principalmente, à utilização de touros com libido excelente (escores 9 e 10), resultado semelhante ao obtido por Pineda et al. (2000a). A taxa média de gestação após a estação de monta de 90 dias foi de 74,69\%, semelhante à observada por Santos et al. (2000), que, estudando a eficiência reprodutiva de touros Nelore submetidos a quatro proporções touro:vaca (1:25, 1:50, 1:75 e 1:100), verificaram taxas médias de gestação de 42,1; 66,5 e 71,0\% aos 30, 60 e 90 dias de estação de monta, respectivamente. Este resultado foi inferior ao mencionado por Costa e Silva et al. (1993), que, utilizando proporção média touro:vaca de 1:37 e 1:54, verificaram taxas de gestação de 91,4 e 94,2\% 
aos 90 dias de estação de monta, e por Fonseca et al. (1997b), que, trabalhando com proporção touro:vaca de 1:40 e 1:60, observaram taxas de gestação de 87,5 e $95,0 \%$ aos 90 dias de estação de monta, respectivamente.

A taxa de gestação observada nesta pesquisa foi relativamente baixa, possivelmente em virtude da utilização de fêmeas em início do período pós-parto (inferior a 35 dias), inclusive houve algumas parições no período da estação de monta. A taxa de gestação final na estação de monta apresentou variação $(\mathrm{P}<0,05)$ entre touros (Tabela 3$)$. A taxa de gestação entre as fêmeas cobertas pelo touro 1 foi maior $(94,23 \%)$, enquanto as taxas obtidas com os touros $2(82,93 \%)$ e $9(84,21 \%)$ não diferiram $(\mathrm{P}<0,05)$. Os touros 6, 7 e 8 foram utilizados em monta múltipla, portanto, não foram ranqueados (Tabela 3), enquanto o touro 12 foi submetido a lote de repasse (inseminação artificial).

A regressão do escore da libido nos períodos de observação apresentou comportamento quadrático $(\mathrm{P}<0,05)$. A classificação atribuída aos touros, segundo os escores obtidos pelos testes da libido a campo, no final de cada hora, foi considerada baixa. O escore da libido variou de acordo com o horário de observação $(\mathrm{P}<0,05)$ e apresentou pequeno aumento nas três primeiras horas de teste, com tendência a estabilização nas duas horas seguintes, diminuindo de forma gradativa após este período e chegando a escore médio de 0,2 (questionável) no horário das 17 às $18 \mathrm{~h}$ (Tabela 4).
No entanto, a classificação atribuída aos touros nos testes da libido a campo de forma acumulativa, no período de 12 horas de observação, elevou o escore da libido para excelente. O escore da libido variou de acordo com o horário de observação $(\mathrm{P}<0,05)$ e aumentou de forma quase linear nas sete primeiras horas de teste, com estabilização na hora seguinte, chegando a escore médio de 10,0 (excelente) no período das 14 às $15 \mathrm{~h}$ (Tabela 4).

Durante o período de 3 às $7 \mathrm{~h}$ de teste, observou-se elevação do escore da libido, provavelmente em virtude do aumento da freqüência dos comportamentos précopulatórios. Isso indica que o tempo inferior a uma hora de teste não é suficiente para avaliar e ranquear adequadamente os reprodutores e que é necessário maior tempo de teste.

O número de animais estudados certamente reduz a confiabilidade dos resultados obtidos, mas não os invalidam, principalmente por terem sido obtidos em animais de rebanhos selecionados e aptos à reprodução, de acordo com exame andrológico e serviços realizados em estações reprodutivas anteriores. Resultado semelhante foi mencionado por Oliveira e al. (2007), que, trabalhando com touros Nelore submetidos ao teste da libido a campo, observaram a necessidade de tempo superior ao preconizado pelo Colégio Brasileiro de Reprodução Animal (CBRA, 1998) para os touros identificarem as fêmeas em estro. Esses autores relataram ainda que nenhum dos touros efetuou

Tabela 3 - Taxa de gestação de fêmeas Nelore submetidas a monta natural em manejo individual por período médio de 90 dias

\begin{tabular}{|c|c|c|c|c|c|c|c|c|c|c|c|c|}
\hline Touro & Gestantes (\%) & Não-gestantes (\%) & Total & & & & $X^{2}$ & & & & & \\
\hline 1 & $49(94,23)$ & $3(5,77)$ & 52 & a & & & & & & & & \\
\hline 2 & $34(82,93)$ & $7(17,07)$ & 41 & $\mathrm{a}$ & c & & & & & & & \\
\hline 3 & $29(65,91)$ & $15(34,09)$ & 44 & b & c & $\mathrm{e}$ & & & & & & \\
\hline 4 & $35(74,47)$ & $12(25,53)$ & 47 & b & c & e & $\mathrm{f}$ & & & & & \\
\hline 5 & $33(67,35)$ & $16(32,65)$ & 49 & $\mathrm{~b}$ & c & e & $\mathrm{f}$ & g & & & & \\
\hline 9 & $32(84,21)$ & $6(15,79)$ & 38 & a & c & e & $\mathrm{f}$ & g & $\mathrm{h}$ & & & \\
\hline 10 & $13(56,52)$ & $10(43,48)$ & 23 & $\mathrm{~b}$ & $\mathrm{~d}$ & e & $\mathrm{f}$ & g & $\mathrm{i}$ & $\mathrm{j}$ & & \\
\hline 11 & $31(77,50)$ & $9(22,50)$ & 40 & $\mathrm{~b}$ & c & e & $\mathrm{f}$ & g & $\mathrm{h}$ & $\mathrm{j}$ & $\mathrm{k}$ & \\
\hline 13 & $21(75,00)$ & $7(25,00)$ & 28 & $\mathrm{~b}$ & c & e & $\mathrm{f}$ & g & $\mathrm{h}$ & $\mathrm{j}$ & $\mathrm{k}$ & 1 \\
\hline 14 & $30(61,22)$ & $19(38,78)$ & 49 & b & $\mathrm{d}$ & e & $\mathrm{f}$ & g & $\mathrm{i}$ & $\mathrm{j}$ & $\mathrm{k}$ & 1 \\
\hline Total & $307(74,69)$ & $104(25,30)$ & 411 & & & & & & & & & \\
\hline
\end{tabular}

a,... valores seguidos por letras diferentes na mesma coluna diferem entre si $(\mathrm{P}<0,05)$ pelo teste qui-quadrado.

Tabela 4 - Médias do escore da libido de touros Nelore avaliado ao final de cada período de observação (12 horas) e por avaliação cumulativa

\begin{tabular}{|c|c|c|c|c|c|c|c|c|c|c|c|c|}
\hline \multirow[t]{2}{*}{ Escore } & \multicolumn{11}{|c|}{ Horário de avaliação } & \multirow[b]{2}{*}{$17-18 \mathrm{~h}$} \\
\hline & 6-7 h & $7-8 \mathrm{~h}$ & 8-9 h & $9-10 \mathrm{~h}$ & $10-11 \mathrm{~h}$ & $11-12 \mathrm{~h}$ & $12-13 \mathrm{~h}$ & $13-14 \mathrm{~h}$ & $14-15 \mathrm{~h}$ & $15-16 \mathrm{~h}$ & $16-17 \mathrm{~h}$ & \\
\hline $\begin{array}{l}\text { Medição ao final } \\
\text { de cada período }\end{array}$ & 3,39 & 4,2 & 4,79 & 5,16 & 5,31 & 5,24 & 4,95 & 4,44 & 3,71 & 2,76 & 1,59 & 0,20 \\
\hline $\begin{array}{l}\text { Avaliação } \\
\text { cumulativa }\end{array}$ & 3,23 & 4,78 & 4,13 & 7,29 & 8,23 & 9,00 & 9,56 & 9,93 & 10,00 & 10,00 & 10,00 & 10,00 \\
\hline
\end{tabular}


serviço completo nas duas primeiras horas de teste. $\mathrm{Na}$ primeira hora de teste, $77,8 \%$ dos touros foram classificados como questionáveis e 22,2\% como bons, enquanto, ao final do teste (13 horas), 11,1\% foram classificados como questionáveis, $22,2 \%$ como bons, $11,1 \%$ como muito bons e $55,6 \%$ como excelentes.

Santos et al. (2004b), avaliando o comportamento sexual de touros Nelore em curral, verificaram que o aumento do tempo do teste da libido, de 10 para 15 minutos, acarretou redução no percentual de touros com libido questionável (de 32,1 para 26,8\%) e com libido boa (de 42,9 para 25,0\%), mas elevou o percentual de touros com libido muito boa (de 21,4 para 42,8\%) e excelente (de 3,6 para 5,4\%). Em outro estudo, Oliveira et al. (2007), observando touros da raça Nelore submetidos ao teste da libido em curral, constataram que, com o aumento no tempo de avaliação, os escores da libido dos touros aumentaram, resultando em melhor classificação. Dessa forma, o aumento no tempo de avaliação, de 10 para 180 minutos, proporcionou redução no percentual de touros com libido questionável (de 76,92 para 15,38\%) e aumentou o percentual de touros com libido excelente (de 0,00 para $76,92 \%)$.

O desconhecimento do desempenho sexual (teste da libido a campo) e dos fatores que podem influenciar o teste é atribuído, em parte, aos poucos estudos nessa área. Assim, novos métodos de avaliação da libido devem ser desenvolvidos, pois o método utilizado neste estudo foi de difícil execução e exige algumas modificações, principalmente no tempo de observação e nas condições de manejo a que os animais foram submetidos.

O tempo para o teste da libido foi muito longo e pode ser considerado inviável e inadequado para a avaliação de grande número de animais em fazendas de pecuária de corte. Além disso, foram verificados problemas na realização do rodeio entre os lotes, uma vez que os piquetes apresentavam tamanhos variados e distantes uns dos outros. A metodologia preconizada pelo Colégio Brasileiro de Reprodução Animal (CBRA, 1998) para avaliar o comportamento sexual de touros zebuínos requer reformulações e mostrou-se ineficiente na classificação dos comportamentos pré-copulatórios como, por exemplo, o reflexo de monta (RM).

O método utilizado para avaliar o comportamento sexual dos touros da raça Nelore seguiu as recomendações preconizadas por Pineda et al. (1997), porém a classificação do escore da libido foi realizada pelo conjunto de eventos fisiológicos no período de uma hora de observação e foi repetido de hora em hora durante todo o período de teste (12 horas).

\section{Conclusões}

A classificação andrológica por pontos e o teste de libido a campo não são eficientes para predizer o potencial reprodutivo de touros da raça Nelore em relação à taxa de gestação ao final da estação de monta, com duração média de 90 dias. O tempo de uma hora de teste da libido não é suficiente para avaliar e ranquear adequadamente os reprodutores.

\section{Agradecimentos}

À Agropecuária CFM Ltda - Fazenda São Francisco Magda, São Paulo, pela colaboração durante a realização deste trabalho.

\section{Literatura Citada}

ALTMANN, J. Observational study of behaviour. Behaviour, v.49, n.1, p.227-267, 1974.

ANDRADE, V.J.; SALVADOR, D.F.; VALE FILHO, V.R. et al. Perfil andrológico de touros da raça Nelore de dois e três anos de idade, criados extensivamente em condições do Estado de Minas Gerais do Mato Grosso do Sul. Revista Brasileira de Reprodução Animal, v.25, n.2, p.182-184, 2001.

ANUALPEC 2003. Anuário estatístico da produção animal. São Paulo: FNP, 2003. 400p.

BLOM, E. Pathological condictions in the genital organs and in the semen of group for rejection of breeding bulls for import or export to and from Denmark, 1958-1982. Nordisk Veterinaer Medicin, v.35, n.3, p.105-130, 1983.

COLÉGIO BRASILEIRO DE REPRODUÇÃO ANIMAL - CBRA. Manual para exame andrológico e avaliação do sêmen animal. 2.ed. Belo Horizonte: 1998. p.49.

CHENOWETH, P.J.; BALL, L. Breeding soundness evaluation in bulls. In: MORROW, D.A. (Ed.) Current therapy in theriogenology. Philadelphia: Saunders Company: 1980 , p.330-339.

CHENOWETH, P.J. Sexual behavior of the bull: a review. Journal of Dairy Science, v.66, n.1, p.173-179, 1983.

COSTA E SILVA, E.V. Capacidade reprodutiva de touros Nelore: exame andrológico, teste de comportamento sexual e desafio de fertilidade. 1994. 102f. Dissertação (Mestrado em Medicina Veterinária) - Escola de Veterinária da Universidade Federal de Minas Gerais, Belo Horizonte, 1994.

COSTA E SILVA, E.V. Comportamento sexual de touros Nelore. In: REUNIÃO ANUAL DA SOCIEDADE BRASILEIRA DE ZOOTECniA, 41., 2004, Campo Grande. Anais... Campo Grande: Sociedade Brasileira de Zootecnia, 2004. p.468-482.

COSTA E SILVA, E.V.; CRUDELI, G.A.; FONSECA, V.O. et al. Comportamiento sexual de toros Nelore (Bos taurus indicus): Efecto de la capacidad de servicio sobre la tasa de fertilidad del rodeo. Cabia, v.1, n.21, p.20-26, 1990.

COSTA E SILVA, E.V.; FONSECA, V.O.; HERMANY, A. et al. Avaliação andrológica de touros Nelore e aptidão reprodutiva: taxa de gestação. Revista Brasileira de Reprodução Animal, v.17, n.3/4, p.97-109, 1993.

FONSECA, V.O. O touro no contexto da eficiência reprodutiva do rebanho. Informe Agropecuário, v.21, n.205, p.48-63, 2000.

FONSECA, V.O.; CRUDELI, G.A.; COSTA E SILVA, E.V. et al. Potencial reprodutivo de touros da raça Nelore (Bos taurus indicus) em monta natural: proporção touro:vaca 1:40 e 
fertilidade. Revista Brasileira de Reprodução Animal, v.15, n.1/2, p.103-108, 1991.

FONSECA, V.O.; VALE FILHO, N.R.; MIES FILHO, A. et al. Procedimentos para exame andrológico e avaliação de sêmen animal. Belo Horizonte: Colégio Brasileiro de Reprodução Animal, 1992, 79p.

FONSECA, V.O.; SANTOS, N.R.; MALINSKI, P.R. Classificação andrológica de touros zebus (Bos taurus indicus) com base no perímetro escrotal e características morfo-físicas do sêmen. Revista Brasileira de Reprodução Animal, v.21, n.2, p.36-39, 1997a.

FONSECA, V.O.; FRANCO, C.S.; BERGMANN, J.A.G. et al. Potencial reprodutivo de touros da raça Nelore (Bos taurus indicus) acasalados com elevado número de vacas. Arquivo Brasileiro de Medicina Veterinária e Zootecnia, v.49, n.1, p.53-62, 1997b.

FRENEAU, G.E.; DIAS, F.M.G.N.; PUOLI, J.R. Avaliação de uma população de touros Nelore pelo índice de capacidade andrológica por pontos (CAP). In: REUNIÃO ANUAL DA SOCIEDADE BRASILEIRA DE ZOOTECNIA, 33., 1996. Anais... Fortaleza: Sociedade Brasileira de Zootecnia, 1996, p.344-347.

FRENEAU, G.E.; PUOLI, J.R.; DIAS, F.M.G.N. Capacidade andrológica por pontos em touros Nelore: resultados após quatro anos de aplicação em MS. In: REUNIÃO ANUAL DA SOCIEDADE BRASILEIRA DE ZOOTECNIA, 35., 1998. Anais... Botucatu: Sociedade Brasileira de Zootecnia, 1998, p.149-151.

FRENEAU, G.E.; PUOLI, J.R.; BORJA, A.L.R. Índice de capacidade andrológica por pontos (ICAP) em touros Nelore: estudo de estação de acasalamento em Mato Grosso do Sul. In: REUNIÃO DA SOCIEDADE BRASILEIRA DE ZOOTECNIA, 37., 2000, Viçosa, MG. Anais... Viçosa, MG: Sociedade Brasileira de Zootecnia, [2000]. (CD-ROM).

GALVANI, F. Desempenho reprodutivo de touros de alta libido da raça Nelore. 1998. 69f. Dissertação (Mestrado em Veterinária) - Universidade Federal de Viçosa, Viçosa, MG, 1998.

GOTTSCHALL, C.S.; MATTOS, R.C. Achados de exames andrológicos em touros de corte Bos taurus e Bos indicus. Revista Brasileira de Reprodução Animal, v.21, n.4, p.25-28, 1997.

HANCOCK, K.L. The morphology of boar spermatozoa. Journal of Royal Microscopical Society, v.76, p.84-97, 1957.

INSTITUTO BRASILEIRO DE GEOGRAFIA E ESTATÍSTICA IBGE. [2009]. Pesquisa Pecuária Municipal (PPM) 2006 a 2007, SIDRA - Sistema IBGE de Recuperação Automática: Quantidade de Animais - Unidades. Disponível em: <http:/ / www.ibge.org.br> Acesso em: 11/3/2009.

LÔBO, R.B. Programa de melhoramento genético da raça Nelore. 4.ed. Ribeirão Preto: Associação Nacional de Criadores e Pesquisadores, 2001. 46p.

OLIVEIRA, C.B.; GUIMARÃES, J.D.; COSTA, E.P. et al. Avaliação do comportamento sexual em touros Nelore: comparação entre os testes da libido em curral e do comportamento sexual a campo. Revista Brasileira de Zootecnia, v.36, n.1, p.32-42, 2007.

PINEDA, N.R.; LEMOS, P.F. Contribuição ao estudo da influência da libido e capacidade de serviço sobre a taxa de concepção em Nelore. Boletim da Indústria Animal, v.51, n.1, p.6168, 1994.

PINEDA, N.R.; LEMOS, P.F.; FONSECA, V.O. Comparação entre dois testes de avaliação do comportamento sexual (libido) de touros Nelore (Bos taurus indicus). Revista Brasileira de Reprodução Animal, v.21, n.4, p.29-34, 1997.
PINEDA, N.R.; FONSECA, V.O.; ALBUQUERQUE, L.G. Estudo preliminar da influência do perímetro escrotal sobre a libido em touros jovens da raça Nelore. Arquivo Brasileiro de Medicina Veterinária e Zootecnia, v.52, n.1, p.69-75, 2000a.

PINEDA, N.R.; FONSECA, V.O.; PROENÇA, R.V. Potencial reprodutivo de touros Nelore: libido, capacidade de serviço e eficiência em acasalamento com elevada proporção de vacas. Revista Brasileira de Reprodução Animal, v.24, n.1, p.44-51, 2000b.

QUIRINO, C.R. Relação fenotípica entre características físicas do sêmen, perímetro escrotal e libido em touros Nelore. In: ENCONTRO DE PESQUISA DA ESCOLA DE VETERINÁRIA DA UNIVERSIDADE FEDERAL DE MINAS GERAIS, 16., 1998, Belo Horizonte. Anais... Belo Horizonte: Escola de Veterinária - Universidade Federal de Minas Gerais, 1998. v.16, p.33.

RIBEIRO FILHO, A.L.; ANDRADE, V. J.; VALE FILHO, V. R. et al. Classificação andrológica por pontos de touros Nelore registrados. In: ENCONTRO DE PESQUISA DA ESCOLA DE VETERINÁRIA DA UNIVERSIDADE FEDERAL DE MINAS GERAIS, 16., 1998, Belo Horizonte. Anais... Belo Horizonte: Escola de Veterinária - Universidade Federal de Minas Gerais, 1998. v.16, p.35.

SALVADOR, D.F.; ANDRADE, V.J.; VALE FILHO, V.R. et al. Desempenho reprodutivo de touros da raça Nelore, submetidos à classificação andrológica por pontos (CAP), à libido e desafiados com alto número de fêmeas com estro sincronizado. Revista Brasileira de Reprodução Animal, v.25, n.2, p.185-187, 2001.

SANTOS, M.D.; TORRES, C.A.A.; RUAS, J.R.M. et al. Potencial reprodutivo de touros da raça Nelore submetidos a diferentes proporções touro:vaca. Arquivo Brasileiro de Medicina Veterinária e Zootecnia, v.56, n.4, p.497-503, 2004a.

SANTOS, N.R.; TORRES, C.A.A.; RUAS, J.R.M. et al. Teste da libido e atividade de monta natural em touros da raça Nelore. Arquivo Brasileiro de Medicina Veterinária e Zootecnia, v.56, n.4, p.504-510, 2004b.

SANTOS, M.D.; TORRES, C.A.A.; RUAS, J.R.M. et al. Potencial reprodutivo de touros da raça Nelore em monta natural submetidos às proporções touro:vaca 1:25, 1:50, 1:75 e 1:100. In: REUNIÃO ANUAL SOCIEDADE BRASILEIRA DE ZOOTECNIA, 37. 2000, Viçosa, MG. Anais... Viçosa, MG: Sociedade Brasileira de Zootecnia. [2000]. (CD-ROM).

SARREIRO, L.C.; QUIRINO, C.R.; PINEDA, J.A.G. et al. Associações genéticas entre libido, perímetro escrotal e qualidade do sêmen de tourinhos da raça Nelore. In: SIMPÓSIO BRASILEIRO DE MELHORAMENTO ANIMAL, 3., 2000, Belo Horizonte. Anais... Belo Horizonte: Simpósio Brasileiro de Melhoramento Animal, 2000. p.449-451.

SARREIRO, L.C.; BERGMANN, J.A.G.; QUIRINO, C.R. et al. Herdabilidade e correlação genética entre perímetro escrotal, libido e características seminais de touros Nelore. Arquivo Brasileiro de Medicina Veterinária e Zootecnia, v.54, n.6, p.602-608, 2002.

SERENO, J.R.B. [2004]. Utilização racional de touros em monta natural. Disponível em: <http://www.agronline.com.br> Acesso em: 10/5/2007.

UNIVERSIDADE FEDERAL DE VIÇOSA - UFV. Sistemas de Análises Estatísticas e Genéticas - SAEG Versão 8.0. Viçosa, MG, 2000. 142p. 